\title{
Erratum: Low-frequency charge noise in Si/SiGe quantum dots [Phys. Rev. B 100, 165305 (2019)]
}

\author{
Elliot J. Connors ๑, JJ Nelson, Haifeng Qiao, Lisa F. Edge, and John M. Nichol
}

(Received 29 June 2020; published 16 July 2020)

DOI: 10.1103/PhysRevB.102.039902

We discovered some errors in our paper. Correcting these errors yields minor changes to some of the figures in the paper as we describe below.

(1) The values of $\gamma(1 \mathrm{~Hz})$ used to generate Fig. 3(b) were incorrectly obtained by fitting the measured noise spectra to a function of the form $S_{\epsilon}(f)=\frac{A}{f^{\beta}}$. Figure E1 shows the correct distribution of exponents, which was obtained by fitting the measured noise spectra to a function of the form $S_{\epsilon}(f)=\frac{A}{f^{\beta}}+\frac{B}{\frac{f^{2}}{f_{0}^{2}}+1}$ as stated in the text. The values of $\gamma(1 \mathrm{~Hz})$ displayed in Fig. 4 and Supplemental Material Fig. 2 were obtained with the correct fit function.

(2) There were typographical errors in the code used to produce the Dutta-Horn (DH) simulations in Fig. 4 and Supplemental Material Fig. 2. The affected parts are the DH simulations of $S_{\epsilon}(1 \mathrm{~Hz})$ (the black lines) in Figs. 4(a)-4(c) and Supplemental Material Figs. 2(a), 2(b), 2(e), 2(f), 2(i), 2(j), 2(m), 2(n), 2(q), 2(r), 2(u), and 2(v). Figures E2 and E3 are the correct version of these figures.

(3) The data of Fig. 4(f) and Supplemental Material Figs. 2(d), 2(h), 2(l), 2(p), 2(t), and 2(x) were incorrectly displayed by averaging the measured values of $\gamma(1 \mathrm{~Hz})$ over $50-\mathrm{mK}$ temperature intervals as opposed to $25-\mathrm{mK}$ temperature intervals as stated in the caption. The corrected figures are displayed with $25-\mathrm{mK}$ averaging.

There are no changes to the text of the paper or the Supplemental Material. The corrected Fig. 4 and Supplemental Material Fig. 2 show the same level of agreement between the calculated and the measured values of $S_{\epsilon}(1 \mathrm{~Hz})$ as the original versions. Therefore, these corrections do not affect the conclusions of the paper.

We thank Professor J. Kestner for bringing some of these errors to our attention.
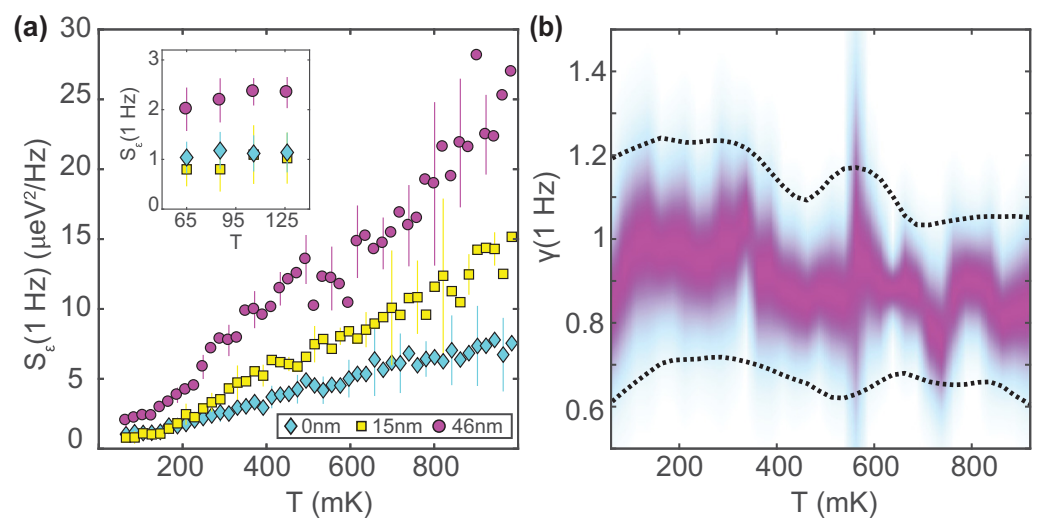

FIG. E1. The correct Fig. 3. 

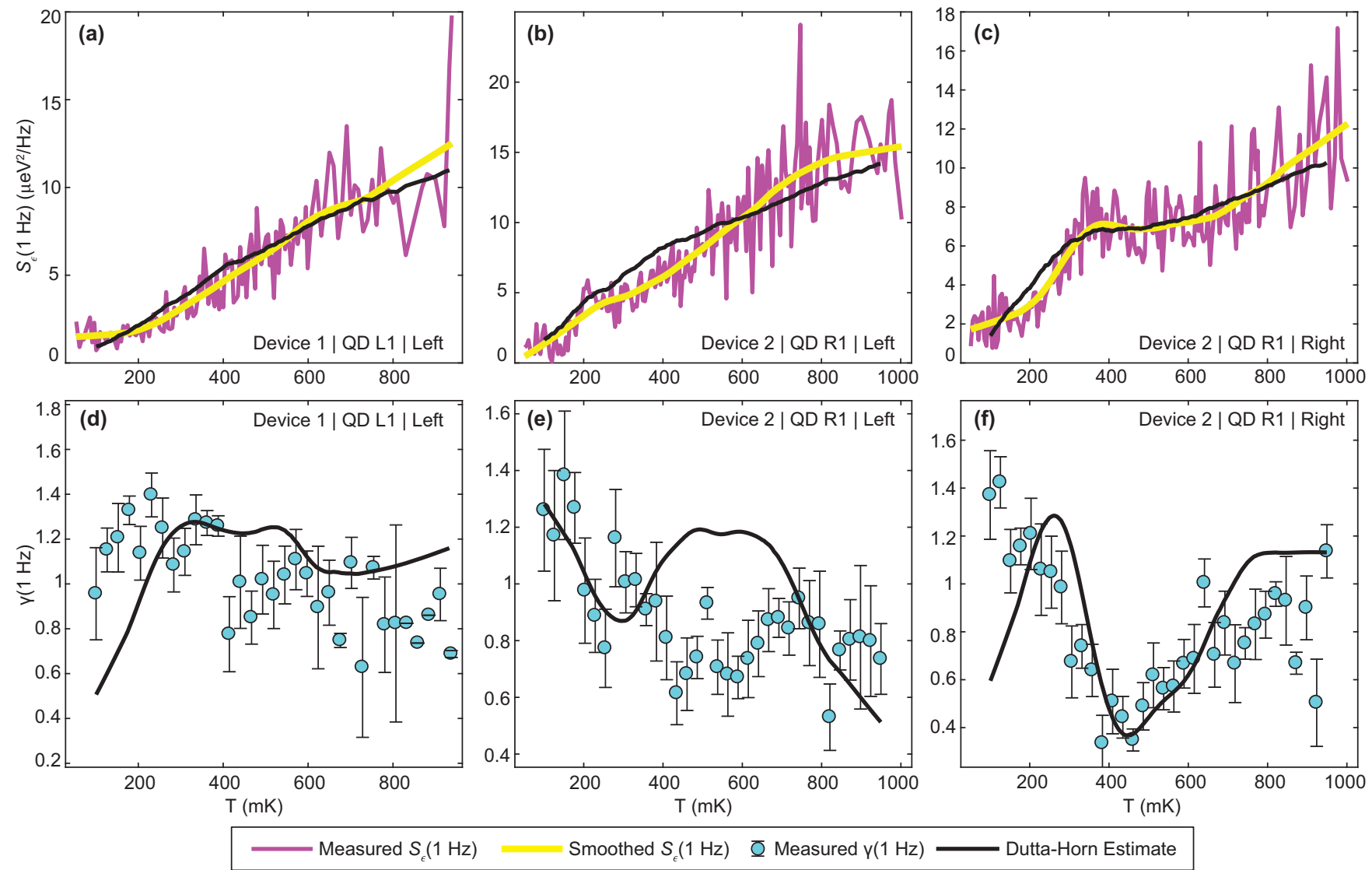

Smoothed $S_{\epsilon}(1 \mathrm{~Hz}) \quad \bar{\varnothing}$ Measured $\gamma(1 \mathrm{~Hz})$

Dutta-Horn Estimate

FIG. E2. The correct Fig. 4. 

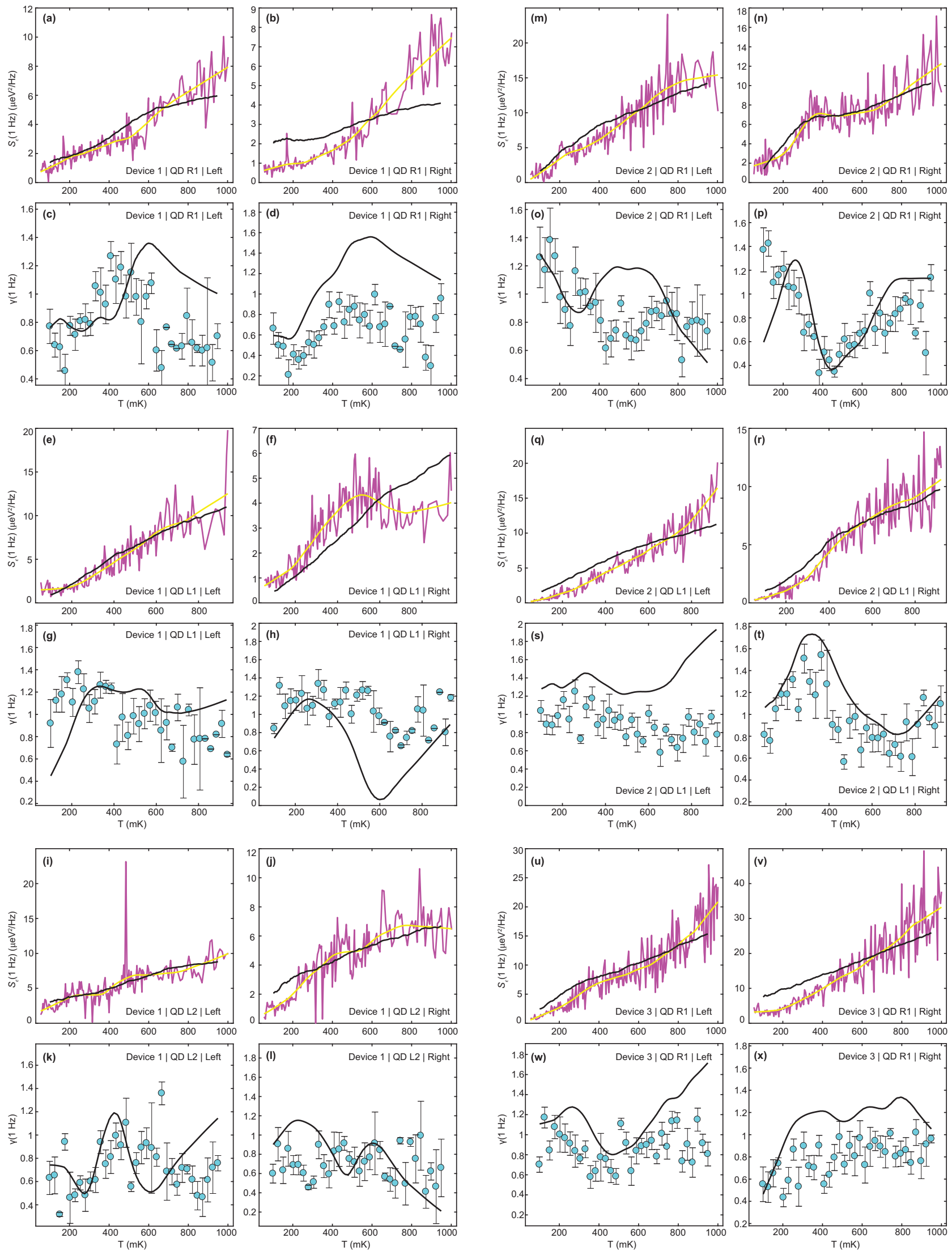

Measured $S_{\epsilon}(1 \mathrm{~Hz}) \quad$ Smoothed $S_{\epsilon}(1 \mathrm{~Hz}) \quad$ $\mathrm{Q}$ Measured $\mathrm{Y}(1 \mathrm{~Hz}) \longrightarrow$ Dutta-Horn Estimate

FIG. E3. The correct Supplemental Material Fig. 2. 